\title{
Environmental Scientiae
}

Jun a Nov $2020-$ v.2 - n.2

\section{Análisis de sistemas ambientales}

Una evaluación de impacto debe abarcar los siguientes aspectos: describirla acción propuesta, así como otras alternativas; predecir la naturaleza y magnitud de los 'efectos ambientales'; predecir los aspectos humanos; interpretar los resultados y prevenir los efectos ambientales. Además, hay que disponer de una metodología para las fases de comunicación (informacional público y al ejecutivo) y, de acuerdo con el caso, para los procedimientos de inspección durante la fase de construcción y de operación del proyecto o la acción de que se trate.

Palabras clave: Inspección; Efectos ambientales; Sistemas ambientales; Metodología.

\section{Análise de sistemas ambientais}

Uma avaliação de impacto deve abranger os seguintes aspectos: descrever a ação proposta, bem como outras alternativas; prever a natureza e magnitude do 'efeitos ambientais'; prever aspectos humanos; interpretar os resultados e prevenir os efeitos ambientais. Além disso, deve estar disponível uma metodologia para as fases de comunicação (informação ao público e ao executivo) e, conforme o caso, para os procedimentos de fiscalização durante a fase de construção e operação do empreendimento ou ação em causa.

Palavras-chave: Inspeção; Efeitos ambientais; Sistemas ambientais; Metodologia.

Topic: Notas Científicas

Reviewed anonymously in the process of blind peer
Received: $15 / 07 / 2020$

Approved: $\mathbf{2 0 / 1 0 / 2 0 2 0}$
Cleber Vinicius Vitorio da Silva (iD)

Universidade Federal Rural do Rio de Janeiro, Brasil

http://lattes.cnpq.br/4275890458575782

http://orcid.org/0000-0001-8337-9615

clebervitorio88@gmail.com

Josimar Ribeiro de Almeida (iD

Universidade do Estado do Rio de Janeiro, Brasi

http://lattes.cnpq.br/3215586187698472

http://orcid.org/0000-0001-5993-0665

almeida@poli.ufri.br

Lais Alencar de Aguiar (iD

Comissão Nacional de Energia Nuclear, Brasil

http://lattes.cnpq.br/5785500333245448

http://orcid.org/0000-0002-1551-4085

aguiar.lais@gmail.com

\section{Pauli Adriano de Almada Garcia (it \\ Universidade Federal Fluminense, Brasil \\ http://lattes.cnpq.br/3866888351512590 \\ http://orcid.org/0000-0002-3934-4332 \\ pauliadriano@gmail.com \\ Vanessa da Silva Garcia (i) \\ Universidade Federal Fluminense, Brasil \\ http://lattes.cnpq.br/2206038698250045 \\ http://orcid.org/0000-0002-7841-8410 \\ vanessa.sgarcia@gmail.com}

\section{Referencing this:}

SILVA, C. V. V.; ALMEIDA, J. R.; AGUIAR, L. A.; GARCIA, P. A. A.; GARCIA V. S.. Análisis de sistemas ambientales. Environmental Scientiae, v.2, n.2, p.39-45, 2020. DOI: http://doi.org/10.6008/CBPC2674$\underline{6492.2020 .002 .0004}$

DOI: $10.6008 / C B P C 2674-6492.2020 .002 .0004$ 


\section{INTRODUCCIÓN}

El interés masivo por los temas ambientales surgió al final de los años sesenta. Fue entonces cuando se empezaron a percibir con nitidez los problemas de deterioro del medio ambiente, que en principio se centraron especialmente en la contaminación. El concepto medio ambiente tuvo e esa época dos acepciones bastante claras, según lo que se aplicara en los países industrializados o en los países en vías de desarrollo. En los primeros, la temática ambiental se concentraba casi exclusivamente en los aspectos de contaminación, en cuyo caso resultaba correcto considerar que los problemas ambientales tenían un carácter tecnológico. En cambio, en los países en vías de desarrollo, el concepto era mucho más amplio y de carácter socioeconómico y político, más que tecnológico, puesto que se consideraban problemas ambientales prioritarios, precisamente los derivados del subdesarrollo: problemas sanitarios, condiciones de los asentamientos humanos, falta de viviendas y escuelas, deficiencias de nutrición, de destrucción de bosques y pérdidas de suelos, destrucción o mala explotación de recursos naturales. A estos problemas del subdesarrollo hay que añadir los que pueden generarse de un desarrollo que no considere en sus proyectos la variable ambiental. Hoy en día, en todos los países, el concepto de medio ambiente tiene un sentido único y generalizado, cada vez más amplio, hasta el punto de que conceptos tan complejos y extensos como los de la calidad de vida y asentamientos humanos se integran en su temática. Persiste sin embargo la idea de que los problemas ambientales en los países industrializados derivan en su mayor parte de procesos de desarrollo y, muy especialmente, de las grandes concentraciones humanas y/o industriales (la contaminación, la deshumanización de la vida en las grandes ciudades, la creciente conflictividad social de las metrópolis, el consumo exagerado de recursos naturales y de energía, los problemas urbanísticos, etc.), en cuanto que en los países en vías de desarrollo los problemas ambientales se deben, principalmente, al bajo índice de crecimiento. Las evaluaciones de impacto ambiental nacieron en los Estados Unidos, como consecuencia de la Ley Nacional de Política Ambiental. Es en ese país donde se han hecho más trabajos de este tipo y, por consiguiente, donde se han desarrollado más metodologías para los mismos. Hasta la fecha se han preparado muchas, pero ninguna de ellas tiene una dimensión o un carácter universal. Así pues, aunque existe gran cantidad de modelos, son pocos los que están sistematizados. La primera evaluación ambiental en Brasil fue realizada en 1972, una exigencia del Banco Mundial para el financiamiento de una represa y de una hidroeléctrica en Sobradinho, Bahia. Otros proyectos como el de la hidroeléctrica de Tucuruí, en Paraná, y el del terminal del puerto ferroviario Ponta de Madeira, en Maranhão, punto de exportación del minerio extraido por la CVRD, en Serra dos Carajás fueron subordinados a la AIA en la década de 70 e inicio de la década de 80 (MOREIRA, 1989).

\section{REPORTE}

Se dice que hay un impacto ambiental cuando una acción o actividad produce una alteración en el medio o en alguno de los componentes del medio. Por lo tanto, la variable fundamental en estos estudios es la cuantificación de la alteración. En los estudios de impacto ambiental se trata de evaluar las consecuencias 
de una acción, para ver la calidad del ambiente que habría con o sin dicha acción. Tales evaluaciones deben realizarse en la fase previa al proyecto, antes que este se realice, con objeto de: a) efectuar una mejor planificación y formulación de propuestas, desde el punto de vista ambiental, y b) considerar adecuadamente los factores ambientales, por parte de las autoridades públicas, cuando aprueben una propuesta o determinen una alternativa. Se aplica el concepto de evaluación del impacto ambiental a un estudio encaminado a identificar e interpretar, así como a prevenir, las consecuencias o los efectos que acciones o proyectos determinados pueden causar a la salud y al bienestar humanos y al entorno, o sea, en los ecosistemas en que el hombre vive y de los que depende (ALMEIDA et al., 2019). La dificultad para demostrar la complejidad de la dinámica ambiental en una única definición hace que, en general, todas las definiciones adquieran un carácter reduccionista y estático. Esto constituye, de cierto modo, el gran problema de las conceptualizaciones. Otras dificultades encontradas en la definición y, especialmente, en la identificación de un impacto ambiental consisten en la propia delimitación del impacto, una vez que el mismo se propaga espacial y temporalmente a través de una compleja red de interrelaciones y, también, en las deficiencias instrumentales y metodológicas para prever las respuestas de los ecosistemas a las actividades antrópicas. Esta cuestión es todavía más crítica cuando se trata de la dimensión social. Los estudios deben considerar las alternativas de la acción y del proyecto. Éstos también presuponen la participación del público representando, no solamente un instrumento de decisión, sino también un instrumento de conocimiento al servicio de la decisión. Existen innumerables definiciones en la literatura especializada del proceso e evaluación de impactos ambientales. La mayoría es de origen académica, enfatizando aspectos técnicos. Otras dan énfasis a los componentes políticos y de gestión ambiental. Existen también las definiciones legales, como la instituida en el NEPA. Para ser útil, la evaluación debe ser comunicada en términos comprensibles para la comunidad y para los responsables por la toma de decisión. Los pros y contras deben ser identificados en base a criterios relevantes para los países afectados. Es una actividad destinada a identificar y prever el impacto sobre el ambiente biogeofísico y sobre la salud y el bienestar de los seres humanos, resultante de propuestas legislativas, políticas, programas y proyectos y de sus procesos operacionales y de interpretar y comunicar las informaciones sobre estos impactos. El término "estimativa ambiental" describe la técnica y el proceso por el cual se colecta información acerca de los efectos ambientales de un proyecto, tanto los producidos por el que lo desarrolla como los generados por otras fuentes. Debe considerarse, inclusive, si el desarrollo amerita seguir enfrente o no, por medio de juicio formado, gracias a la autoridad profesional. Instrumento de política ambiental, formado por un conjunto de procedimientos, capaz de asegurar, desde el inicio del proceso, que se haga un examen sistemático de los impactos ambientales de una acción propuesta (proyecto, programa, plan o política) y de sus alternativas, y que los resultados sean presentados de forma adecuada al público y a los responsables por la toma de decisión, y por ellos considerados. Además de eso, los procedimientos deben garantizar la adopción de las medidas de protección del medio ambiente determinadas, en el caso de decisión sobre la implantación del proyecto (ALMEIDA et al., 2019). Una estimativa del impacto de una actividad planeada en el ambiente. En suma, la evaluación de impactos ambientales es "un instrumento de política ambiental, formado por un 
conjunto de procedimientos, capaz de asegurar, desde el inicio del proceso, que se haga un examen sistemático de los mismos en una acción propuesta (proyecto, programa, plan o política) y de sus alternativas. Además, que los resultados sean presentados de forma adecuada al público y a los responsables por la toma de decisiones, debidamente considerados por éstos" (ALMEIDA et al., 2019). Según este autor, las definiciones identifican importantes y distintos componentes. Uno de ellos es el que engloba un conjunto de procedimientos para identificar, evaluar y prevenir efectos adversos y que debe estar relacionado con conocimiento científico sobre el ambiente, la acción y sus interrelaciones. El otro componente es el proceso de toma de decisión, en el cual la evaluación de impactos de una acción puede tener un importante papel que está íntimamente relacionado con reglas administrativas y voluntad política. En la literatura de habla inglesa se adoptan términos, como 'Environmental Impact Assessment' (EIA) para designar estudios que engloban conjuntamente aspectos sociales y ecológicos, y 'Ecological Impact Assessment' y 'Social Impact Assessment' para los que tratan de aspectos ecológicos y sociales, respectivamente. Un término que engloba mejor esos estudios es el 'Integrated Impact Assessment', que se refiere al estudio del conjunto de consecuencias sociales y ecológicas según un enfoque holístico, que ponga en evidencia los efectos cumulativos resultantes.

\section{DISCUSIÓN}

Es importante resaltar que la investigación guiada por el método dialéctico revela la historicidad del fenómeno y sus relaciones, a un nivel más amplio, sitúan el problema dentro de un contexto complejo, al mismo tiempo, estableciendo y señalando las posibles contradicciones entre los fenómenos investigados. La investigación cualitativa se basa en la inseparabilidad de los fenómenos y su contexto, ya que las opiniones, percepciones y significados se entenderán con mayor profundidad desde el contexto. La validez se referiría a la similitud entre el concepto y sus medidas, el grado en que una medida representa precisamente lo que se espera. La garantía de validez comenzaría con una comprensión directa de lo que se debe medir, por lo tanto, se trata de formulación de la investigación. El método propuesto se dividió en tres fases, que se subdividieron en etapas para la realización del relevamiento bibliográfico. En la primera fase de la investigación (preguntas de investigación), se verifican las principales decisiones y definiciones sobre la investigación. Las preguntas de investigación se destacan y sirven como impulso para el inicio de la revisión. Se define el tema para definir la encuesta bibliográfica y el período disponible. Otras decisiones se toman simultáneamente, como definir palabras clave, para que actúen como identificadores. Paralelamente, resumen los principales temas sobre el tema a investigar; así como combinaciones de palabras clave. Estos, a su vez, se pueden realizar mediante operadores booleanos y en diferentes bases de datos como CAPES Journal Portal, SCIELO Database, Publish or Perish, EBSCO, entre otras. En el presente caso, las bases de datos fueron Web of Science (o ISI), Plataforma SUSTENERE, Scielo y Scopus. Estas bases de datos son de fácil acceso, lo que permite, a través de sus herramientas, realizar una cuidadosa investigación, abarcando un amplio número de revistas. La segunda fase (selección de artículos) inicia la encuesta y selección de artículos, utilizando los criterios de exclusión. Las palabras clave de exclusión se utilizan para realizar una clasificación 
más cuidadosa de los artículos. También delimitando el período. En el siguiente paso, se produce el primer filtro de los artículos, se realiza una selección de los títulos, identificando los no alineados. En el siguiente paso, se leen los resúmenes para excluir a aquellos que son impertinentes con el tema investigado. El siguiente paso contiene un análisis subjetivo por parte del investigador para identificar, en los artículos alineados con la temática, aquellos que tienen mayor relevancia académica. Un análisis bibliométrico indica la relevancia del autor/artículo para la composición de la referencia bibliográfica. La tercera fase corresponde a la clasificación de los artículos seleccionados. Se organizan muestras de artículos seleccionados. El objetivo es el orden, patrón que variará según las necesidades del investigador y lo priorice como foco de desarrollo temático. Destaca la importancia de un análisis exhaustivo de los artículos que componen la muestra. En la investigación cualitativa, el concepto de validez toma diferentes formas, ya que la discusión de las escalas de medición no se aplica a los métodos cualitativos, lo que requiere una comprensión de la validez desde otra perspectiva. Un atributo que se relaciona con la objetividad, la posibilidad de repetir el experimento, el hecho de que la investigación esté abierta a la verificación de otras personas y la capacidad de generalizar (SOUZA et al., 2011). La validez puede verse genéricamente como la correspondencia entre investigación y realidad (MONTANDON et al., 2015). Se refiere a verificar los resultados como verdaderos y confiables. Estaría relacionado con el hecho de que los resultados reflejan fielmente la situación analizada y son confiables, en el sentido de que no habría razón para dudar de ellos; es decir, la investigación es válida si la evidencia proporciona el soporte necesario para sus conclusiones (LINS et al., 2015). La intención no es generalizar, sino describir, analizar, buscar comprender.

El empleo de análisis de sistemas permite evaluar las interacciones que ocurren cuando una actividad humana se desarrolla, afectando tanto la naturaleza como los aspectos sociales y económicos. La metodología utilizada para evaluación de los impactos, por ejemplo, de una terminal petroquímica situada en una cuenca del Estado del Paraná (Brasil), se basa en un modelo de situación ambiental en la región de aquella terminal. Se consideró una región como un sistema (o sea, un conjunto de factores físicos, humanos y económicos relacionados formando una unidad dinámica) compuesto de varios sistemas (sociales, económicos y naturales) relacionados entre sí.

\section{Etapas}

Identificación de los límites del sistema: Todo sistema posee límites arbitrarios. Actualmente, los límites terrestres presentan un radio de $2 \mathrm{Km}$ alrededor de la terminal y el marítimo presentan área, dependiendo de las direcciones de las corrientes marinas, con una extensión de $5 \mathrm{Km}$;

Definición de la Escala: La escala definida es la local, o sea, de la terminal petroquímica- región urbana vecina - terminal - región de la cuenca contigua al terminal;

Influencia de las entradas ('inputs') y salidas ('outputs') del sistema: Las entradas son representadas por el aporte de productos químicos, através de camiones y navíos, y las salidas son representadas por los cargamentos de camiones y navíos con productos guardados en la terminal, así como por los fluidos producidos en la terminal (aguas pluviales contaminadas o no, vías de desagüe y lanzamientos de productos 
químicos);

Identificación de los componentes o subsistemas: Subsistema económico (productos químicos, energía eléctrica, agua, camiones, navíos), Subsistema social (urbanización, empleo) y Subsistema natural (suelos, água subterránea, vegetación, ecosistemade la cuenca).

\section{Objetivos del modelo}

La definición de un sistema trata, básicamente, de establecer una simplificación y abstracción de la realidad (en este caso, local), permitiendo la identificación de elementos específicos que componen el sistema. Para el sistema ambiental del área de la terminal, los elementos identificados representan tanto su entorno como los flujos de materiales hacia o desde la terminal.

\section{Evaluacion de impacto ambiental sobre cada sector}

Economía regional: Evaluación de las consecuencias sobre la economía regional, o sea, ¿en qué contribuirá la terminal al sistema de exportación e importación de los productos químicos por el Estado de Paraná (Brasil)? ¿Qué efectos tendrán los posibles impactos negativos sobre el aumento previsto de exportación e importación de productos químicos generados por la terminal?;

Salud pública: Evaluación de las consecuencias de la operación de la terminal y del aumento de la importación y exportación de productos químicos, debido a la terminal, sobre la salud de la población;

Calidad del aire: Evaluación de las consecuencias de operación y de accidentes en la terminal sobre la calidad del aire;

Calidad del suelo: Evaluación de las consecuencias de operación y de accidentes en la terminal sobre la calidad del suelo;

Calidad del agua: Evaluación de las consecuencias de la operación y de accidentes en la terminal sobre la calidad del agua subterránea y de la cuenca;

Ecosistemas: Evaluación de las consecuencias de la operación y de accidentes en la terminal sobre los variados elementos del ecosistema y sobre el ecosistema en general.

\section{CONSIDERACIONES FINALES}

Las consecuencias deben ser inseridas en un cuadro, de modo que sean colocadas a la izquierda las consecuencias positivas y a la derecha las negativas. A continuación, caso a caso ( $p$. ej. salud pública $x$ desagües sanitarios), se debe verificar:

Si hay relación entre sector y consecuencias; Evaluar si el impacto es importante o muy importante e indicar respectivamente, con uno o dos asteriscos; A continuación, sumar los impactos positivos o negativos y el número total de esos impactos, por ejemplo:

№ de interacciones posibles $=400$ № de interacciones existentes $=37 №$ de interacciones positivas $=$ $17 №$ de interacciones negativas $=204$.

Calcular el índice de interacciones, para cada subsistema, por la expresión: 


$$
I I=\frac{R}{I P}
$$

Donde:

$\|$ = índice de interacción

$I R=$ interacciones posibles

$I P=$ interacciones positivas

Si el valor del índice fuese igual a 1 (uno), la posibilidad de ocurrencia de interacciones positivas o sea, ausencia de impactos negativos, será de 100\%; El índice de interacciones representa las posibilidades de impactos en cada subsistema y permite, incluso, clasificar esos subsistemas, según el grado y tipo de impactos posibles en cada uno. Se puede, finalmente, para cada subsistema, discutir los impactos negativos y recomendar alternativas y/o medidas.

\section{REFERENCIAS}

ALMEIDA, J. R.; SILVA, C. E. ; SILVA, C. V. V. ; AGUIAR, L. A. ; GARCIA, V. S. ; SOUZA, C. P. ; LENZ, E. R. S. ; LINS, G. A. ; ALMEIDA, S. M.. Política e economia de vigilância em saúde ambiental. Environmental Scientiae, v.1, p.1-25, 2019. DOI: https://doi.org/10.6008/CBPC2674-6492.2019.002.0001

SOUZA, F. M. N.; SILVA, C. E.; AGUIAR, L. A.; ALMEIDA, J. R.. Proposta para utilização da simulação computacional em análise de risco, avaliação de desempenho e sistemas de gestão ambiental. Revista Ibero-Americana de Ciências Ambientais, v.2, p.39-63, 2011. DOI: https://doi.org/10.6008/ESS2179-6858.2011.002.0003
MONTANDON, T. S.; CAMELLO, T. C. F.; ALMEIDA, J. R. Indicadores de sustentabilidade para monitoramento de projetos de recuperação de áreas degradadas. Sustinere: Revista de Saúde e Educação, v.3, p.43-52, 2015. DOI: http://dx.doi.org/10.12957/sustinere.2015.17326

LINS, G. A.; BEZERRA, L. G. E.; MOTA, M. J. P.; BARBOSA, O. R.; ALMEIDA, J. R.. A ecologia de estrada sob a ótica do licenciamento ambiental. Sustinere: Revista de Saúde e Educação, v.3, p.153-160, 2015. DOI:

http://dx.doi.org/10.12957/sustinere.2015.20143 